\title{
Primary squamous cell carcinoma of the caecum: case report and update
}

\begin{abstract}
First described in 1919, primary squamous cell carcinoma (SCC) of the large bowel is an extremely rare colonic malignancy that appears to arise preferentially within the caecum. The underlying aetiology remains unclear, although it may involve chronic inflammation leading to glandular-squamous metaplasia. Here we present the case of a young male patient who presented following an episode of collapse secondary to an acute-gastrointestinal bleed, who was subsequently found to have metastatic SCC of the caecum. Whereas the survival from colorectal adenocarcinoma has improved dramatically over the previous 100 -years, prognosis from caecal SCC remains very poor
\end{abstract}

Keywords: squamous cell carcinoma, SCC, caecum, colorectal cancer
Volume 5 Issue 8 - 2016

\section{Mason JD, Teranaka W, Freebairn A, Kingston G, Middleton SB}

Department of Surgery, Royal Berkshire Hospital, UK

Correspondence: John Mason, Department of Surgery, Royal Berkshire Hospital, Craven Road, Reading, RGI 5AN MRCS, UK, Email masonjd85@gmail.com

Received: October 26, 2016 | Published: December 29, 2016

\section{Background}

The glandular structure of the colorectal mucosa means that approximately $90 \%$ of colonic cancers are adenocarcinoma. The remaining $10 \%$ of tumours consist of neuroendocrine, adenosquamous, spindle cell, undifferentiated carcinomas and lymphoma. ${ }^{1}$ Pure squamous cell carcinoma (SCC) of the colon is extremely rare, estimated to account for less than $0.025 \%$ of all colonic malignancies. ${ }^{2}$

The first case of primary colorectal SCC localised to the caecum was described in 1919 by Schmidtmann, ${ }^{3}$ followed by Raiford. ${ }^{4}$ who described a case arising within the rectum. SCC of the rectum is more common than caecal SCC, arising within close proximity to the anorectal transition zone (ATZ), ${ }^{5}$ where mixed glandular and squamous cell populations coexist. Therefore, there is debate as to whether this subgroup of cancers should be classified as adenosquamous or pure SCC. Colorectal SCC is associated with poor prognosis, although individuals with rectal disease tend to survive longer than those with caecal SCC. ${ }^{6}$ This may be explained by anatomical differences, whereby rectal SCC is more likely to provide recognisable symptoms (fresh PR bleeding and/or luminal obstruction) at an early stage when surgical resection (with or without neoadjuvant radiotherapy) followed by adjuvant chemotherapy are feasible.

Here we present the case of a 28 -year-old male with an acute presentation of PR bleeding secondary to a previously undiagnosed primary SCC of the caecum. This is accompanied by a review of the literature to date.

\section{Case report}

A 28-year-old male patient presented following an episode of collapse while playing football with an associated unprovoked PR bleed. There was no previous history of PR bleeding and the blood was dark/clotted, although not typical of melena. Coinciding with this was a 6-month episode of generalised abdominal pain, worse in the right iliac fossa (RIF) with associated weight loss, although he had not sought medical attention for this as it did not pose any limitations on his day-to-day lifestyle. He took regular NSAIDs for musculoskeletal pain, although there was no prior history of haematemesis. He reported no other medical or surgical history and there was no family history of relevant gastrointestinal (GI) or any other systemic diseases.
On examination he had an anaemic appearance but was haemodynamically stable. Abdominal examination revealed some tenderness in the lower abdomen, worse in the RIF, although no obvious masses or organomegaly were palpable. There was no peritonism. PR examination revealed some clotted blood on the glove.

Initial full blood count demonstrated a normocytic anaemia consistent with an acute GI bleed (Hb 95g/L, MCV 83.5fL). White cell count was normal, although $\mathrm{C}$-reactive protein was elevated at $114 \mathrm{mg} / \mathrm{L}$. Liver function tests showed an isolated raised ALT $(121 \mathrm{IU} / \mathrm{L})$. Iron studies demonstrated features consistent with iron deficiency anaemia, despite a normal MCV (iron 2umol/L, transferrin $1.5 \mathrm{~g} / \mathrm{L}$, transferrin saturation $5 \%$ and ferritin $792 \mathrm{ug} / \mathrm{L}$ ).

As it was unclear whether the PR bleeding had an upper or lowerGI aetiology an initial oesophago-gastro-duodenoscopy (OGD) was undertaken, which was normal. Flexible sigmoidoscopy showed a rectum full of altered blood and histological examination of biopsies from the descending colon, sigmoid colon and rectum all demonstrated the presence of scattered macrophages throughout the lamina propria (without granulomas), with an associated alteration in the normal gradient of inflammatory cells and active lymphoid aggregates, suggestive of idiopathic inflammatory bowel disease (IBD). There were no features of dysplasia of malignancy.

He then underwent full colonoscopic examination, which revealed a large friable and bleeding mass within the caecum (Figure 1). There were no macroscopic areas of ulceration elsewhere within the colon. Histology of the caecal mass reported a moderately differentiated squamous cell carcinoma (Figure 2A). Subsequent immunohistochemistry analysis showed that the tumour cells were strongly positive for CK5/6 and p63, but CK20 and CDX2 negative, consistent with a diagnosis SCC (Figure 2B). Coinciding with this, tumour markers were found to be elevated (CEA 16ug/L).

A full staging CT-scan confirmed the presence of a large caecal mass with adjacent, mesenteric and para-aortic lymph nodes measuring up to $1.5 \mathrm{~cm}$. There were also several low attenuation lesions within the liver, confirmed to be metastatic disease on a subsequent MRI scan. There was no evidence of disease within the chest on this initial scan. However, a subsequent whole body PET CT-scan showed FDGavid and CT-enlarged lymphadenopathy within the retroperitoneum 
and above the diaphragm within the posterior mediastinum and left supraclavicular fossa. The caecal tumour was characterised as an $8.4 \mathrm{~cm}$ FDG-avid mass arising from the caecum with an area of central necrosis. Multiple (over 10) liver metastases affecting both lobes of the liver were also demonstrated.

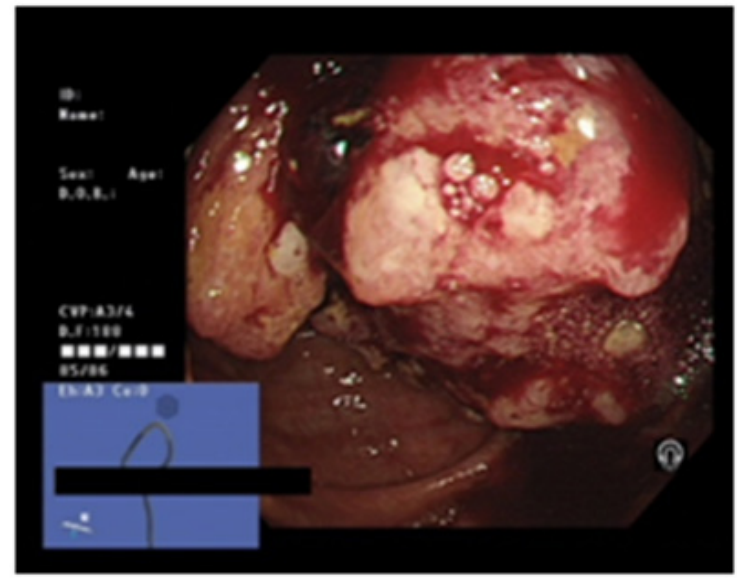

Figure I Colonoscopy image of friable caecal mass.

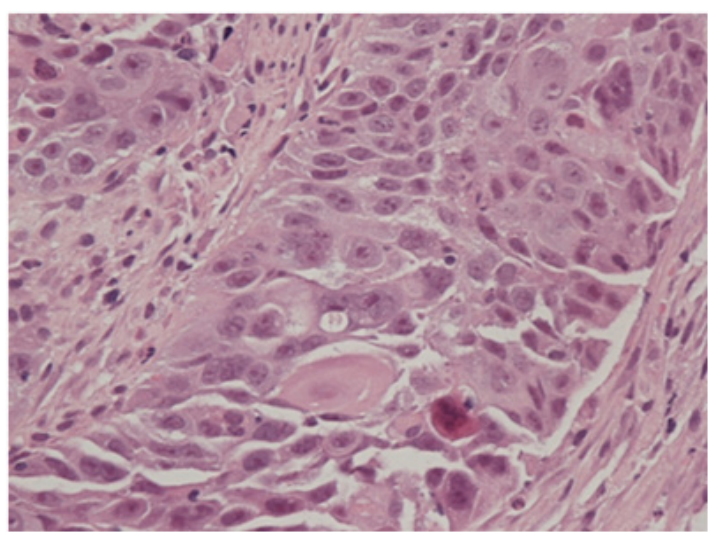

Figure 2A Haematoxylin and eosin staining (x200 magnification) demonstrating neoplastic squamous epithelium characterised by sheets of cohesive epithelioid cells with enlarged, irregular, ovoid nuclei and prominent nucleoli as well as eosinophilic cytoplasm. Squamous differentiation is demonstrated in the form of intercellular bridges and the presence of keratinised cells.

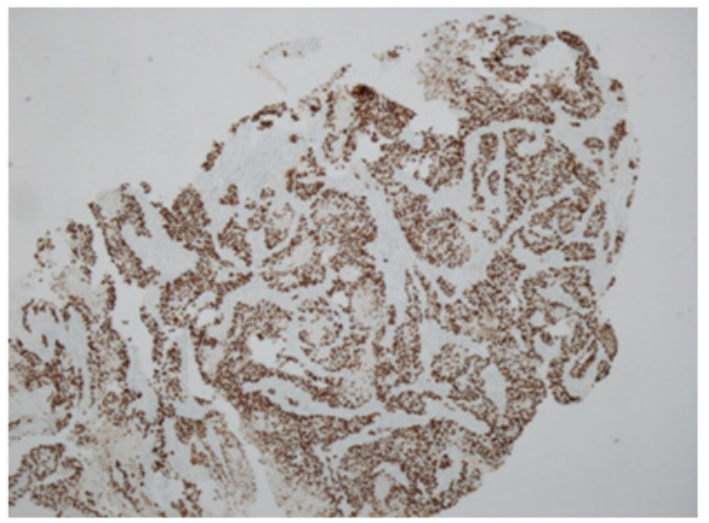

Figure 2B p63 immunohistochemistry (x40 magnification) demonstrating diffuse nuclear p63 immunoexpression within tumour cells, which is typical of SCC and absent in adenocarcinoma.
Following discussion at the colorectal multi-disciplinary team (MDT) meeting a diagnosis of metastatic primary SCC of the caecum was made. Given the advanced stage without evidence of impending large bowel obstruction surgical treatment was not deemed to be appropriate. Following discussion with the patient and his family, a palliative treatment plan was adopted, including a trial of cisplatin and 5-flurouracil (5'-FU) based palliative chemotherapy. Unfortunately, a re-staging scan following 3-cycles of chemotherapy showed disease progression.

\section{Discussion}

First described by Schmidtmann. ${ }^{3}$ in 1919 primary SCC of the caecum is a rare colorectal malignancy with less than 20 cases reported in the literature. Rectal SCC is more common, although whether these cases reflect pure SCC or adenosquamous disease arising within close proximity to the anorectal junction is debatable. Diagnosis of primary colorectal SCC requires three parameters to be excluded: absence of a non-colonic primary SCC, exclusion of an underlying squamous-lined fistula to the tumour site and exclusion of anal SCC extending into the rectum. ${ }^{7}$ Application of this diagnostic criteria shows that while SCC may arise within the rectum, there are no reports of pure SCC arising in any other part of the colon except for the caecum. ${ }^{8}$

SCC of the caecum and rectum probably share common aetiological factors, including chronic inflammation from infection and/or inflammatory bowel disease (IBD). Infection with human papillomavirus (HPV) has been shown to be associated with rectal SCC, ${ }^{9}$ and concomitant infection with schistosomiasis has been reported in caecal SCC..$^{10}$ The role of chronic inflammation in driving caecal SCC tumour development was first described by Lewis et al., ${ }^{11}$ who hypothesised that SCCs arise from embryonic nests of undifferentiated epithelium experiencing a chronic inflammatory stimulus. This theory is supported by modern day understanding of tumour development within a stem cell niche originating from the mucosal crypts. ${ }^{12}$ An alternative mechanism may involve epidermoid implantation during surgery. ${ }^{11}$ Indeed, evidence for this process is provided by Balsano, ${ }^{13}$ who describes two cases of caecal SCC in patients who had undergone appendicectomy and right inguinal hernia repair complicated by post-operative chronic sinus and enterocutaneous fistula formation. The association between IBD and SCC occurring within the rectum is well reported, ${ }^{8,14}$ although there has only been a single case of caecal SCC arising in patient with longstanding ulcerative colitis (UC) ${ }^{15}$ Interestingly, this case also had a co-existing neuroendocrine tumour, which has also been described in a case report of rectal SCC. ${ }^{16}$ Cases of metachronous bowel tumours arising in patients with primary colorectal SCC may indicate an underlying genetic component. While the patient within this case was not previously known to have IBD, all non-tumour mucosal biopsies demonstrated evidence of IBD.

Consistent with this case, presenting symptoms of primary caecal SCC appear to resemble known features of caecal adenocarcinoma. Previously reported cases primary SCC of the caecum commonly list non-specific lower abdominal pain, weight loss and anaemia as the main presenting features. ${ }^{8,13,17,18}$ There appears to be a male predominance in patients presenting with primary SCC of the caecum, with over half of the published case reports occurring in young menalthough Kulaylat et al. ${ }^{8}$ observed the opposite when rectal SCC arises in patients with coexisting $\mathrm{UC}$, which is more common in females. Of interest, the patient in this case study was found to be anaemic $(\mathrm{Hb}$ $95 \mathrm{~g} / \mathrm{L})$, although he had a normal MCV $(83.5 \mathrm{fL})$. The full extent of 
his iron deficiency anaemia was revealed on subsequent iron studies. This highlights an important observation that iron deficiency anaemia can arise with a normal MCV - a finding that is observed in up to $40 \%$ of individuals with pathological anaemia. ${ }^{19}$

Survival from colorectal cancer has improved dramatically over recent decades. However, survival from primary SCC of the caecum remains very poor, and has not improved much since 1919 despite the dramatic improvements seen in surgical and (neo) adjuvant therapies. Typically, survival is less than 1-year from presentation, with apparent resistance to chemotherapeutic agents (cisplatin and 5'-FU) known to be effective in non-colonic SCCs. This may be explained by the fact that the majority of cases in the literature are occurring in young patients with non-specific symptoms meaning diagnosis is often delayed until the disease is at an advanced metastatic stage. . $^{17,18,20,21}$ In contrast, survival from primary rectal SCC is more favourable, probably explained by the fact that these patients often have coexisting IBD meaning that they will undergo surveillance, and even in the absence of IBD these patients are more likely to report symptoms such as PR bleeding, which may prompt earlier investigation. This enables aggressive oncological treatment with surgical resection and adjuvant chemotherapy, meaning that survival in patients with lymph node negative disease is over 2years (range: $2-21$ years) ${ }^{8}$

In summary, SCC of the caecum is a rare disease, and usually affects young male patients. It often presents at an advanced stage with poor prognosis ( $<1$ year following presentation). It is a distinct disease from adenosquamous cell tumours that arise close to the recto-anal junction. There appears to be an association with chronic inflammation, which promotes tumour development and drives progression. Treatment with evidence-based SCC sensitive chemotherapy appears to have limited therapeutic effect.

\section{Acknowledgment}

None.

\section{Conflicts of interest}

Authors declare that there are no conflicts of interest.

\section{Funding}

None.

\section{References}

1. Donghee Kim, Su Yeon- Coi, Eun Ha Park, et al. Nonalcoholic fatty liver disease is associated with coronary artery calcification. Hepatology. 2012;56(2):605-613.

2. Philip Noel Newsome, Allison ME, Andrews PA, et al. Guidelines for liver transplantation for patients with non-alcoholic steatohepatitis. Gut. 2012;61(4):484-500.

3. Pinzani. Exploring beyond cirrhosis. Hepatology. 2012;56(2):778-780.

4. Li Chen, Rui Chen, Hua Wang, et al. Mechanisms Linking Inflammation to Insulin Resistance. Int J Endocrinol. 2015;2015:508409.

5. Kento Imajo, Takaomi Kessoku, Yasushi Honda, et al. Magnetic Resonance Imaging More Accurately Classifies Steatosis and Fibrosis in fatty liver disease than Transient Elastography. Gastroenterology. 2016;150(3):626-637.

6. Angela M Zivkovic, J Bruce German, Arun J Sanyal. Comparative review of diets for the metabolic syndrome: implications for nonalcoholic fatty liver disease. Am J Clin Nutr. 2007;86(2):285-300.

7. Hussain TA, Mathew TC, Dashti AA, et al. Effect of low-calorie versus low-carbohydrate ketogenic diet in type 2diabetes. Nutrition. 2012;28(10):1016-1021.

8. Filozof C, Goldstein BJ, Williams RN, et al. Non-Alcoholic Steatohepatitis: Limited Available Treatment Options but Promising Drugs in Development and Recent Progress Towards a Regulatory Approval Pathway. Drugs. 2015;75(12):1373-1392. 